\title{
Mental health and the impact of ubiquitous technologies
}

\author{
Bert Arnrich · Venet Osmani · Jakob Bardram
}

Published online: 30 September 2011

(C) Springer-Verlag London Limited 2011

\begin{abstract}
This Theme issue focuses on the emerging research of ubiquitous technologies to support mental health. So far, the majority of work presented in the field of ubiquitous healthcare has focused on supporting people affected by somatic diseases. However, increasing number of diseases affecting mental health has prompted research on technologies to support people suffering from these diseases. This Theme issue provides a number of examples of research on the potential impact of ubiquitous technologies in the field of mental health.
\end{abstract}

\section{Introduction}

Up till now, the majority of research within pervasive healthcare has focused on pervasive computing for somatic diseases such as diabetes, hypertension, heart failure, and obesity [1]. Even though some research has been directed toward dementia and autism, less focus has been given to targeting major mental disorders such as chronic stress, depression, anxiety, bipolar disorder, or mania. It is important to note that mental disorders affect around 25\% of all people at some time during their life. Moreover, these disorders are universal-affecting all countries and societies and individuals at all ages. According to the World Health Organization, the negative direct and indirect

B. Arnrich

ETH Zurich, Zurich, Switzerland

V. Osmani ( $\square)$

CREATE-NET, Trento, Italy

e-mail: venet.osmani@create-net.org

J. Bardram

IT University of Copenhagen, Copenhagen, Denmark impact on economy and on the quality of life of individuals and families is massive [17].

Pervasive and ubiquitous computing technologies and approaches can have a positive impact for people affected by mental disorders, since a promising approach is to teach patients to recognize and manage early warning signs on their own. In order to successfully realize this new kind of therapy, patients and therapists need continuous support in assessing early warning signs in an objective and timely way. This is where ubiquitous computing technologies can play a critical role.

\section{Background}

Pervasive healthcare has seen an emergence of a new research strand, focused toward mental health and wellbeing in parallel with other, more traditional somatic diseases. This strand has focused on diseases that affect cognition, such as Alzheimer's, motor neuron diseases such as Parkinson's, developmental disorders such as autism, and more recently mood disorders including depression and bipolar disorders or combination of diseases, and consequent diagnosis of comorbidities.

In the current pervasive healthcare literature, manifestation of cognitive decline is typically measured through monitoring of performance of Activities of Daily Living (ADL). In this respect, The Aware Home, developed by Georgia Institute of Technology [9], is a living laboratory for interdisciplinary research, development, and evaluation. Among numerous projects that are aimed at improving services at home, some of them address assistive technologies for older adults, such as Cook's Collage, Memory Mirror, Digital Family Portrait, and others. Another system aimed to provide better assessment, diagnosis, treatment, and 
evaluation of behavioral problems for the elderly is developed at the Carnegie Mellon University [15]. The authors emphasize the importance of close and continuous monitoring that provides the specialists with better assessment of individual's behavior and allows them for more appropriate interventions. Kaye [7] argues that for the purpose of dementia prevention, it is intrinsic to enable the detection of trajectories of change over many years and to get deeper into sensitive domains such as determining the information about increasing variability in patients' behavior.

A number of research works have investigated motor neuron diseases such as Parkinson's patients where wearable assistive technology that allows patients to recover from gait freezing has been investigated [2]. Automated administration of wolf motor function to determine the functional ability of the paretic and non-paretic limbs of post-stroke patients has been researched, and a successful feasibility study has been reported [16]. Monitoring of dressing activity for after-stroke patients and patients who experience difficulties due to cognitive impairment has similarly been investigated [12].

Development disorders have been in focus of pervasive healthcare research for some time. One of the well-known works in this area is by Abowd et al. [8], who describe a system enabling support for caregivers of autistic children. Furthermore, research has been investigating technology to aid autistic adolescents learn to recognize facial expressions [11]. In the same area, work has investigated the link between the use of interactive robots and increased social activity in children with autism spectrum disorder [4].

In the traditional medical research, various studies on correlating mental disease symptoms with objective physiological and behavioral measures have been conducted. Most of these studies were performed in clinical settings and not in the natural environment of the patient. There have been various studies on correlating depression with measures such as skin conductance response, heart rate, and temperature. Research has shown that the autonomic branch of the central nervous system mediates both skin conductance response and heart activity [5]. Patients with depression exhibit an increased baseline heart rate compared with non-depressed subjects [10]. There have been many studies involving continuous monitoring of heart rate rhythms for psychiatric illnesses, which found that depression patients exhibit a rising heart rate pattern during sleep, explaining an early awakening phenomenon that has been observed in these patients [6].

In cognitive behavioral therapy (CBT), software tools were developed to help patients follow a particular therapy [13]. Recently, therapy-related applications that run on handheld devices such as PDAs have appeared, for instance, in the treatment for anxiety and depression [14]. As part of the SCOPE (Supporting Cognition Outlines on
Psychological Evaluation) project, handheld-based tools were developed to let therapists customize CBT to treat anxiety, depression, and associated disorders [3].

Finally, the European Commission has recently called for the development of ICT-based solutions supporting persons suffering from stress, depression, bipolar disorders, or other mental disorders (European Seventh Framework Program ICT-2009.5.1b). The commission asks for interdisciplinary research that addresses the parallel development of technological solutions, as well as new management or treatment models based on closed-loop approaches.

\section{Theme issue}

This Theme issue has selected five papers that deal with a range of mental health disorders, including stress, anxiety, bipolar disorder, and associated technologies, platforms, and services. Each of the papers is briefly presented in the paragraphs that follow.

\subsection{Cross-validation of bimodal health-related stress assessment}

Broek et al. present their research on the development of a voice-based ubiquitous stress assessment. A collective of 25 patients who suffer from post-traumatic stress disorder was investigated. Each patient participated in a storytelling (RT) and in a reliving (RT) session. Both sessions consist of a stress-provoking part and a happy part, respectively. Two instruments were chosen to assess the stress level of the patients at various points in time: (1) patient's speech features and (2) patient's subjective rating according to the Subjective Unit of Distress (SUD) as ground truth measure. A collection of statistical parameters derived from patient's speech features was employed to build 3 independent classifier systems: k-nearest neighbors, support vector machines, and multi-layer perceptron neural network. The discriminative power of the 3-classifier systems was evaluated on 2-10 levels of the SUD rating. As a result, it was shown that between 90 (2 SUD levels) and 39\% (10 SUD levels) correct classification was achieved.

\subsection{Monitoring of mental workload levels during an everyday life office-work scenario}

Cinaz et al. investigate continuous monitoring of physiological signs related to prolonged high workload in order to prevent long-term health damage. The paper presents how mental workload levels in everyday life scenarios can be discriminated with data from a mobile ECG logger by incorporating individual calibration measures. An experimental design is presented that allows to induce three 
different levels of mental workload in a calibration sessions and to monitor mental workload levels in everyday life scenarios. Multiple regression analysis is employed to model the relationship between relevant heart rate variability (HRV) features and the subjective ratings of mental workload levels during office work. In addition, the performance of three classification methods to identify the mental workload level during office work is evaluated.

\subsection{A mobile data collection platform for mental health research}

Gaggioli et al. present the design, development and an initial deployment of a mobile phone platform to collect users' psychological, physiological, and activity information related to mental health. The platform allows collection of self-report questionnaires in parallel with the recording of heart rate and activity information from a wireless sensor system. It is shown how the combination of self-reports with heart rate and activity data enables investigation of the relationship between psychological, physiological, and behavioral variables, as well as monitoring their fluctuations over time.

\subsection{Virtual reality and mobile phones in the treatment for generalized anxiety disorders: an ubiquitous approach phase- 2 clinical trial}

Repetto et al. present a clinical protocol for the treatment of generalized anxiety disorders (GAD) based on the ubiquitous use of a biofeedback-enhanced virtual reality (VR) system. The protocol includes the use of a mobile exposure system allowing patients to perform the virtual experience in an outpatient setting. A between-subjects study, involving 25 GAD patients, was carried out to verify the efficacy of the proposed approach. It is shown that VR is advantageous in the treatment for GAD and that patients appreciated the mobile device.

\subsection{Toward long-term monitoring of electrodermal activity in daily life}

Setz et al. investigate the long-term monitoring of electrodermal activity (EDA) to support the diagnosis and treatment for bipolar disorder patients. In order to realize a long-term monitoring of the EDA, the integration of the sensor system in the shoe or sock is followed. The paper presents a first step toward such a sensor system. In a feasibility study including 8 subjects, the correlation of EDA measurements at the fingers with measurements of the EDA at the feet was investigated. The results indicate that the foot recording location is suitable for recordings in daily life even in the presence of moderate movement.

\section{References}

1. Arnrich B, Mayora O, Bardram J, Tröster G (2010) Pervasive healthcare-paving the way for a pervasive, user-centered and preventive healthcare model. Methods Inf Med 49:67-73

2. Bächlin M, Plotnik M, Roggen D, Giladi N, Hausdorff JM, Tröster G (2010) A wearable system to assist walking of Parkinson's disease patients. Methods Inf Med 49:88-95

3. de Sa M, Carrico L, Antunes P (2007) Ubiquitous psychotherapy. Pervas Comput IEEE 6(1):20-27

4. Feil-Seifer D, Black M, Mataric M, Narayanan S (2009) Toward designing interactive technologies for supporting research in autism spectrum disorders. In: International meeting for autism research

5. Hugdahl K (1995) Psychophysiology: the mind-body perspective. Harvard University Press, Cambridge

6. Iverson G, Gaetz M, Rzempoluck E, McLean P, Linden W, Remick R (2005) A new potential marker for abnormal cardiac physiology in depression. J Behav Med 28:507-511. doi:10.1007/ s10865-005-9022-7

7. Kaye J (2008) Home-based technologies: a new paradigm for conducting dementia prevention trials. Alzheimers Dement 4:60 66

8. Kientz JA, Arriaga RI, Abowd GD (2009) Baby steps: evaluation of a system to support record-keeping for parents of young children. In: CHI'09: proceedings of the 27th international conference on human factors in computing systems. ACM, New York, pp 1713-1722

9. Kientz JA, Patel SN, Jones B, Price ED, Mynatt ED, Abowd GD (2008) The Georgia tech aware home. In: CHI'08: CHI'08 extended abstracts on Human factors in computing systems. ACM, New York, pp 3675-3680

10. Lahmeyer HW, Bellur SN (1987) Cardiac regulation and depression. J Psychiatr Res 21(1):1-6

11. Madsen M, El Kaliouby R, Eckhardt M, Hoque ME, Goodwin MS, Picard R (2009) Lessons from participatory design with adolescents on the autism spectrum. In: CHI'09: proceedings of the 27th international conference extended abstracts on human factors in computing systems. ACM, New York, pp 3835-3840

12. Matic A, Mehta P, Rehg JM, Osmani V, Mayora O (2011) Monitoring dressing activity failures through RIFD and video. J Methods Inform Med 50(4). http://bit.ly/qhRDGx

13. Newman MG (2004) Technology in psychotherapy: an introduction. J Clin Psychol 60:141-145

14. Proudfoot JG (2004) Computer-based treatment for anxiety and depression: is it feasible? Is it effective? Neurosci Biobehav Rev 28:353-363

15. Wactlar H, Hauptmann A, Kanade T, Stevens S, Bharucha A (2007) Caremedia: automated video and sensor analysis for geriatric care. Technical report, Carnegie Mellon University

16. Wade E, Parnandi AR, Mataric MJ (2010) Automated administration of the wolf motor function test for post-stroke assessment. In: 4th international ICST conference on pervasive computing technologies for healthcare

17. WHO (2001) The world health report: 2001: mental health: new understanding, new hope. World Health Organization, Geneva. http://www.who.int/whr/2001/en/ 